\title{
Ultra-Pulsed Radioporation further enhances the efficacy of Dynamic Quadripolar RadioFrequency in women with post-menopausal vulvo-vaginal atrophy
}

\author{
Rosario Tranchini ${ }^{1}$ and Mauro Raichi ${ }^{2 *}$ \\ ${ }^{1}$ Obstetrician and Gynaecologist, Santa Maria di Loreto Nuovo Hospital, Naples, Italy \\ ${ }^{2}$ Clinical Pharmacology and Biophysics Consultant, Milan, Italy
}

\begin{abstract}
Background: A growing body of evidence illustrates the benefits experienced by women with vulvo-vaginal atrophy/genitourinary syndrome of menopause (VVA/ GSM) undergoing vaginal rejuvenation with the very recent low-energy Dynamic Quadripolar RadioFrequency (DQRF ${ }^{\mathrm{TM}}$ ) technology. Twelve-month follow-up data describe significant improvement from both a clinical (relief of VVA/GSM symptoms) and psychological perspective (women's self-esteem and satisfaction from sexual life and couple relationship). The proprietary Ultra-Pulsed Radioporation (UPR ${ }^{\mathrm{TM}}$ ) technology is intended to associate the anti-atrophic benefits of both the DQRF ${ }^{\mathrm{TM}}$ technology and any topical agent with anti-atrophic properties. UPR ${ }^{\mathrm{TM}}$ acts by opening aqueous channels in cell membranes and further modulating DQRF ${ }^{\mathrm{TM}}$ performance. $\mathrm{UPR}^{\mathrm{TM}}$ helps any active principle with useful properties to penetrate the deep layers of vulvar skin and vaginal mucosa. Topical hyaluronic acid (HA) is increasingly used, based on solid biological rationale, to help slow and reverse the menopause-related loss of elasticity and volume of vulvo-vaginal tissues. This pilot study was designed to verify if the novel UPR ${ }^{\mathrm{TM}}$ technology, applied to a test anti-atrophic topical agent like HA in combination with standard DQRF procedures, could indeed enhance the already established anti-atrophic efficacy of the DQRF ${ }^{\mathrm{TM}}$ technology.

Methods: Prospective, randomised, open-label study; two parallel groups of 30 women with evidence of vaginal atrophy and dryness and other postmenopausal VVA/

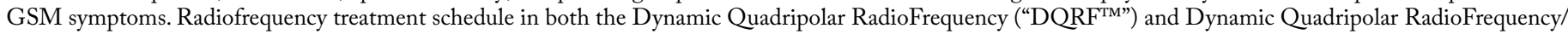
Ultra-Pulsed Radioporation ("DQRF ${ }^{\mathrm{TM}}+\mathrm{UPR}^{\mathrm{TM}}$ ") study arms: five 15-min sessions every 14-16 days following application of either standard or UPR coupling gel. Operative temperatures in target tissues during procedure: $42^{\circ} \mathrm{C}$ (range $40-43^{\circ} \mathrm{C}$ ). Self-administered evaluation tools (before and at the end of the treatment sessions): $10-\mathrm{cm}$ visual analogue scales (VAS) for VVA/GSM symptoms (vaginal dryness, itching and burning, dyspareunia, dysuria/incontinence), 13-item Female Sexual Distress Scale-Revised (FSDS-R) questionnaire (multi-perspective assessment of the woman's personal distress related to sexual dysfunction), Sexual Satisfaction Questionnaire (SSQ) (sexual gratification.) Non-parametric statistical analysis (Wilcoxon Signed Rank and McNemar tests).

Results: On average, all the assessed parameters (VAS symptom scores, FSDS-R and SSQ scores related to the sexual sphere) underwent statistically significant or highly significant improvements in both the "DQRF ${ }^{\mathrm{TM}}+\mathrm{UPR}^{\mathrm{TM}}$ " active group and "DQRF" controls over the about two months of the treatment program. The observed improvements, though always very strong, were somewhat less impressive for at least some parameters in the control "DQRF" ${ }^{\mathrm{TM}}$ " group compared with the active "DQRF" ${ }^{\mathrm{TM}}+\mathrm{UPR}^{\mathrm{TM}}$ " treatment group. That was distinctively the case for vaginal itching, dyspareunia and dysuria/incontinence among VVA/GSM symptoms ("DQRF ${ }^{\mathrm{TM}}+\mathrm{UPR}^{\mathrm{TM}}$ " vs. "DQRF ${ }^{\mathrm{TM}}$ " VAS scores: $-60.9 \%$ vs. $-49.2 \%,-63.8 \%$ vs. $-50.5 \%$, and $-59.3 \%$ vs. $-44.9 \%$, respectively), and for sexual satisfaction from intercourse activity ("DQRF ${ }^{\mathrm{TM}}+\mathrm{UPR}^{\mathrm{TM}}$ " vs. "DQRF"

Discussion: The novel UPR ${ }^{\mathrm{TM}}$ technology was devised to modify the performance of the DQRF ${ }^{\mathrm{TM}} \mathrm{EVA}^{\mathrm{TM}}$ device to facilitate the deep penetration of any topical active principle that has demonstrated to have a favourable impact on the atrophy of female post-menopausal intimate tissues. The idea behind the UPR ${ }^{\mathrm{TM}}$ concept was to enhance the established rejuvenation effect of the DQRF ${ }^{\mathrm{TM}}$ technology thanks to the synergy between the biological effect of the radiofrequency treatment and that of the topical active principle. In the current short-term pilot study, low-molecular weight HA was chosen as the model topical active principle to test the UPR ${ }^{\mathrm{TM}}$ concept. This short-term pilot study confirmed the high vaginal rejuvenation efficacy over a short time of the established DQRF ${ }^{\mathrm{TM}}$ technology in post-menopausal women. Moreover, the study suggested that the novel UPR ${ }^{\mathrm{TM}}$ technology is likely to further enhance the DQRF ${ }^{\mathrm{TM}}$ clinical benefits. Long-term studies are warranted to confirm these preliminary encouraging results.
\end{abstract}

\section{Introduction}

Low-energy Dynamic Quadripolar RadioFrequency $\left(\mathrm{DQRF}^{\mathrm{mx}}\right)$ technology is one of the most recent evolutions in the field of light- and energy-based technologies. DQRF ${ }^{\mathrm{rx}}$ has already shown its potential for vulvo-vaginal rejuvenation in postmenopausal women experiencing vulvo-vaginal atrophy and related symptoms of genitourinary syndrome of menopause (VVA/GSM) with, often, severe disruption of quality of life [1-3]. The proprietary $\mathrm{DQRF}^{\text {six }}$ technology is patented all over the world by Novavision Group S.p.A. (Misinto, Monza-Brianza, Italy). An extensive clinical research programme with the $D Q R F^{m}$ - based device, EVA ${ }^{\mathrm{Tm}}$, is in progress, and follow-up has reached one year with encouraging safety and efficacy data [1].

${ }^{\star}$ Correspondence to: Mauro Raichi, Clinical Pharmacology and Biophysics Consultant, Milan, Italy, E-mail: mraichi@gmail.com

Key words: electroporation, post-menopausal atrophy, radiofrequency, urinary incontinence, vaginal rejuvenation

Received: June 11, 2018; Accepted: June 22, 2018; Published: June 25, 2018 
Tranchini R (2018) Ultra-Pulsed Radioporation further enhances the efficacy of Dynamic Quadripolar RadioFrequency in women with post-menopausal vulvovaginal atrophy

There is some indication from all these data that the new DQRF technology might overcome the problems of low manageability and safety sometimes experienced with other light- and energy-based vulvo-vaginal rejuvenation strategies [3]. At the core of the new hightech EVA ${ }^{\text {tw }}$ device is the $\operatorname{VDR}^{\mathrm{mw}}$ (Vaginal Dynamic Radiofrequency ${ }^{\mathrm{Tw}}$ ) quadripolar 1.0-1.3 $\mathrm{MHz}$ radiofrequency technology. $\mathrm{VDR}^{\text {тx }}$ is based on four stainless steel, electronically controlled dynamic electrodes on anatomical probes with a maximum emitting power of $55 \mathrm{~W}$. All happens within the four radiofrequency electrodes that continuously cycle, under electronic control, between receiver and transmitter states eliminating the need for grounding pads on the upper thigh. In the ideal configuration, the repelling electric fields that are generated concentrate the thermal effect with high tridimensional precision in the target vulvo-vaginal subepithelial layers. This allows respect of surrounding tissues and reduces the administered energy by almost eliminating Ohm's resistances in tissues. Electronically controlled movement and temperature sensors (RSS ${ }^{m}$, Radiofrequency Safety System $^{\mathrm{m}}$ technology) allow rigid control of tissue temperature, eliminating all needs for systemic analgesia or local anaesthesia in the treated area. This area has usually a diameter of some $12 \mathrm{~cm}$, or about 4 inches, centred on the hymenal ring. Women can pause the session at will thanks to a feedback button [3].

A further very recent development of the $\mathrm{DQRF}^{\mathrm{su}}$ concept is the proprietary Ultra-Pulsed Radioporation ${ }^{\mathrm{nw}}\left(\mathrm{UPR}^{\mathrm{Tw}}\right)$ technology. The cue for developing the $\mathrm{UPR}^{\mathrm{ix}}$ technology was taken from radiofrequency electroporation techniques long used in genetic engineering to allow high-efficiency gene transfection and transfer of biological macromolecules into cells [4,5]. UPR ${ }^{\mathrm{m}}$ acts by opening aqueous channels in cell membranes through modulation of the radiofrequency effects of the DQRF ${ }^{\text {rix }}$ technology. This is useful to allow the massive transfer of any active principle with useful properties down to the deep layers of vulvar skin and vaginal mucosa. Speculatively, the effect on post-menopausal vulvo-vaginal hypotrophy and loss of tissue elasticity by $\mathrm{DQRF}^{\mathrm{ru}}$ could sinergise with the anti-atrophic properties of the topical active principles. Always speculatively, the biophysics behind the $\mathrm{UPR}^{\mathrm{Ti}}$ technology could facilitate the efficient penetration of topical active principles into vulvo-vaginal tissues, further enhancing the $\mathrm{DQRF}^{\text {mo }}$ anti-atrophic efficacy.

Cross-linked HA restores the extracellular matrix needed for fibroblast activation and collagen and elastin production [6]. A small yet increasing number of papers over the last few years has investigated the role of hyaluronic acid (HA) to counteract the age-related loss of elasticity and volume of female external genitalia. The aesthetic perspective was dominant in some of these studies, carried out with HA dermal fillers in women with mild to moderate labia majora hypotrophy $[7,8]$. Other studies with topical HA formulations were more focused on the VVA/ GSM symptoms of vaginal dryness, burning and itching, dyspareunia and dysuria/incontinence. Outcomes, both in terms of symptom relief and respected ecology of the vaginal microenvironment, were similar for topical HA and estriol or conjugated oestrogen formulations [9-12].

All these evidences support topical HA as a fine active principle to test the value of the novel UPR ${ }^{\text {in }}$ technology. A coupling gel additioned with low-molecular weight HA (about $290 \mathrm{kDa}$ ) was developed to test in a pilot study whether combining the $\mathrm{DQRF}^{\mathrm{ma}}$ and $\mathrm{UPR}^{\mathrm{mx}}$ technologies could further enhance the established benefits of DQRF $F^{\mathrm{mw}}$ rejuvenation in post-menopausal women.

The herein described double-blind pilot study was designed to compare the short-term evolution of VVA/GSM symptoms and women's gratification and satisfaction with sexual life in two random samples of post-menopausal women randomised to rejuvenation with either the established DQRF $F^{\mathrm{Tw}}$ technology or the novel DQRF ${ }^{\mathrm{TN}}+\mathrm{UPR}^{\mathrm{Tw}}$ approach. In the five planned sessions, a standard coupling gel and the HA-additioned gel were respectively used. The two coupling gels could not be identified.

\section{Materials and methods}

\section{Screening and randomisation}

Sixty VVA/GSM women reporting no menstruation for at least 12 months were screened and randomised, after giving informed consent, between January and July 2017 within the pool of more than 150 outpatients regularly attending a specialist department for post-menopausal disorders in a private clinic. All screened women referred postmenopausal vaginal dryness, evidence of mucosal atrophy (thinning or loss of vaginal rugae, mucosal pallor, etc.), and other VVA/ GSM-related symptoms; an explicit wish for a still active sexual life was also a must. Hormonal replacement therapy, any pelvic organ prolapse beyond the hymenal ring, chronic vulvar pain, vulvar dermatitis or dystrophy, viral lesions, including high risk for human Papillomavirus infections, and poor thickness of the recto-vaginal septum at pelvic examination led to exclusion of candidates. Any active genital or urinary tract infection required treatment before enrolment.

The randomisation log to the two double-blind treatment groups ("DQRF" $F^{\mathrm{m} \text { " }}$ as controls, "DQRF $\mathrm{F}^{\mathrm{mm}}+\mathrm{UPR}^{\mathrm{m \omega} \text { " }}$ as active group; 30 women per group) was generated with the help of a random numbers generator. All study materials, including informed consent forms, study protocol and electronic case report forms, were peer-reviewed for ethical problems and authorised by the clinic authorities. All women gave informed consent to anonymous collection of their data before the first treatment session.

\section{Outcome evaluation}

The clinical severity of VVA/GSM symptoms (vaginal dryness, burning and itching, dyspareunia, dysuria/incontinence) was selfassessed by participants immediately before each of the five treatment sessions using the same $10-\mathrm{cm}$ visual analogue scales (VAS) used in previous DQRF ${ }^{m}$ studies ("No symptom" at the left VAS extreme and "Symptom as severe as it could be" at the right extreme) $[1,3]$. The self-administered Female Sexual Distress Scale-Revised (FSDS-R) questionnaire was used to assess the main factors related to sexual dysfunction affecting the women's personal distress. The FSDS-R responses are based on the frequency with which each problem has caused distress to the woman within the recall periods (for this study, the previous 7 days) [13]. An Italian translation of the Sexual Satisfaction Questionnaire (SSQ - 6-level ordinal responses: none, poor, fair, good, very good, excellent) was also used to evaluate sexual satisfaction from vaginal intercourse. All categorical responses were translated into ordinal scores for statistical analysis (for instance for the SSQ scale, none $=0$, poor $=1$, fair $=2, \ldots$, excellent $=5$ ).

\section{$\mathrm{DQRF}^{\mathrm{m}}$ and $\mathrm{DQRF} \mathrm{F}^{\mathrm{m} /} / \mathrm{UPR}^{\mathrm{mm}}$ treatment protocol}

- Five 15-min sessions, spaced 14-16 days

- Setting of the radiofrequency generator: $1 \mathrm{MHz}$

- Operating power: $25 \%$ of the maximum device power $(55 \mathrm{~W})$

- Target temperature in vulvo-vaginal tissues during procedure: $42^{\circ} \mathrm{C}$ (range $40-43^{\circ} \mathrm{C}$ ) 
Tranchini R (2018) Ultra-Pulsed Radioporation further enhances the efficacy of Dynamic Quadripolar RadioFrequency in women with post-menopausal vulvovaginal atrophy

\section{Operative procedures}

Five treatment sessions were planned spaced 14-16 days. Power was applied for 15 minutes using either the $\mathrm{DQRF}^{\mathrm{mm}}$ or $\mathrm{DQRF}^{\mathrm{mm}}+\mathrm{UPR}^{\mathrm{m}}$ coupling gels starting behind the hymenal ring, with circular back-andforth continuous movements and always keeping contact between the tip probe and the mucosa. The DQRF ${ }^{\text {mi }}$ power was set at $25 \%$ of the device maximum power $(55 \mathrm{~W})$. A standard coupling gel was applied before each DQRF ${ }^{\mathrm{mm}}$-only session to the control women of the "DQRF treatment group. The HA-supplemented coupling gel used in women of the "DQRF $F^{\mathrm{m}}+\mathrm{UPR}^{\mathrm{m} m \text { " }}$ treatment group (combining the established $\mathrm{DQRF}^{\mathrm{mm}}$ and novel UPR ${ }^{\mathrm{mm}}$ technologies) was indistinguishable from the $\mathrm{DQRF}^{\mathrm{ma}}$ coupling gel and was pre-prepared by adding 5 grams of HA to the usual dose of standard coupling gel. Neither the operator nor the treated woman knew which coupling gel was being applied. The operator and the treated woman were similarly unaware if a DQRF ${ }^{\mathrm{m}}$ alone or a $\mathrm{DQRF}^{\mathrm{m}}+\mathrm{UPR}^{\mathrm{mix}}$ modified $\mathrm{EVA}^{\mathrm{Tw}}$ device were being used and all procedures were double blind. Safety, with special attention to pain and discomfort, was assessed in all women at each study visit and by telephone calls over the following days.

\section{Statistical analysis}

Descriptive statistics were generated for demographics and physical examination findings. The nonparametric Wilcoxon Signed Rank Test for repeated measurements on single populations was applied to both repeated measures of ordinal data (converted FSDS-R and SSQ mean scores) and continuous variables (VAS mean scores); the McNemar test was used to test for differences in ordinal scores. The Wilcoxon Signed Rank Test will also be instrumental in the forthcoming morphological assessment. Two-sided 95\% confidence levels were used for all statistical tests with $\mathrm{p}<0.05$ as cut-off for significance.

\section{Results}

All randomised women completed their double-blind treatment program as planned without missing visits. Table 1 illustrates the comparable demographics of the two study groups before the first treatment session. All participant women described their treatment sessions as comfortable; no burns or other complications were reported. All women resumed their everyday and sexual activities immediately after the end of their treatment program.

Figure 1 illustrates the evolution of the VVA/GSM symptoms over the about two months of the "DQRF" $+\mathrm{UPR}^{\mathrm{m} \omega}$ " and "DQRF" programs. On average, all the assessed VVA/GSM symptoms underwent statistically significant or highly significant improvements in both treatment groups. Compared with the basal situation, VAS mean scores were at least halved $(\mathrm{p}<0.01)$ after the end of the treatment program in the "DQRF" $+\mathrm{UPR}^{\mathrm{m} \omega \mathrm{mo}}$ group (vaginal dryness $-59.0 \%$, vaginal itching $-60.9 \%$, vaginal burning $-59.4 \%$, dyspareunia $-63.8 \%$, dysuria/ incontinence $-59.3 \%$ ). The basal mean scores were also at least halved $(\mathrm{p}<0.01)$ in the control group at the end of the "DQRF" program for vaginal dryness $(-51.4 \%)$, vaginal burning $(-59.4 \%)$ and dyspareunia $-50.5 \%)$, but the registered symptomatic improvement was somewhat less for vaginal itching $(-49.2 \%, \mathrm{p}<0.01)$ and dysuria/incontinence $(-44.9 \%, \mathrm{p}<0.05)$. There was no statistically significant difference in the evolution of VVA/GSM symptoms between the "DQRF" $F^{\text {Tx }}+\mathrm{UPR}^{\text {isus" }}$ and the "DQRF" treatment groups for vaginal burning $(-59.4 \%$ in both treatment groups), while there was a borderline non-significant difference for vaginal dryness $(-59.0 \%$ vs. $-51.4 \%, \mathrm{p} \approx 0.06)$, and a highly significant difference $(\mathrm{p}<0.01)$ for vaginal itching $(-60.9 \%$ vs. $-49.2 \%)$, dyspareunia $(-63.8 \%$ vs. $-50.5 \%)$ and dysuria/incontinence $(-59.3 \%$ vs. $-44.9 \%)$.
Table 1. Demographics of the "DQRFTM+UPR ${ }^{\mathrm{TM}}$ " and "DQRF" standard deviation; HRT, hormone replacement therapy

\begin{tabular}{|c|c|}
\hline ACTIVE GROUP (“DQRF+UPR”) & \\
\hline Age (years, mean \pm SD) & $58.1 \pm 5.7$ \\
\hline Body Mass Index $\left(\mathrm{kg} / \mathrm{m}^{2}\right.$, mean \pm SD) & $23.6 \pm 2.5$ \\
\hline Previous live births (n, \%) & $25(83 \%)$ \\
\hline Mean parity (range) & $1.3(1-4)$ \\
\hline Current sexual activity (n, \%) & $25(83 \%)$ \\
\hline Previous HRT (n, \%) & $10(33 \%)$ \\
\hline CONTROL GROUP (“DQRF”) & $57.9 \pm 6.8$ \\
\hline Age (years, mean \pm SD) & $24.2 \pm 3.1$ \\
\hline Body Mass Index (kg/m ${ }^{2}$, mean \pm SD) & $27(90 \%)$ \\
\hline Previous live births (n, \%) & $1.7(1-4)$ \\
\hline Mean parity (range) & $23(77 \%)$ \\
\hline Current sexual activity (n, \%) & $12(40 \%)$ \\
\hline Previous HRT (n, \%) & \\
\hline
\end{tabular}

Sexual dysfunction-related distress before and at the end of the double-blind treatment program

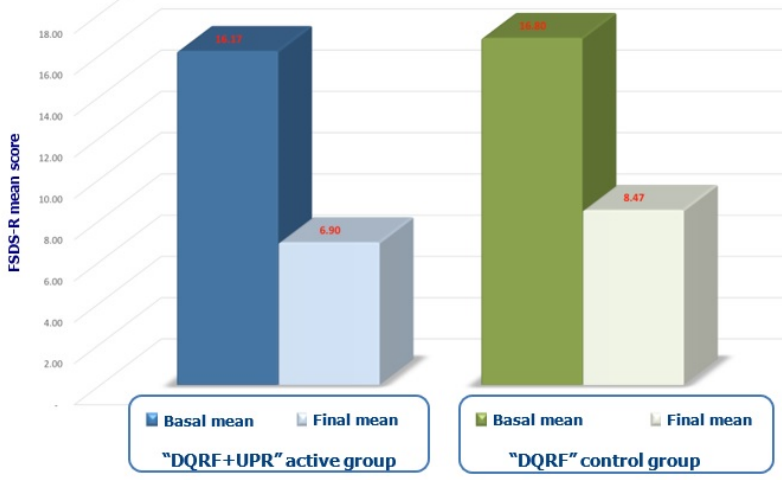

Figure 1. VAS mean scores for the VVA/GSM symptoms in the active "DQRFTM + UPR" treatment group compared with the control "DQRFTM" treatment group

Figure 2 illustrates how the women's personal distress related to sexual dysfunction evolved in the "DQRF ${ }^{\mathrm{rm}}+\mathrm{UPR}^{\mathrm{Tw} \text { " }}$ treatment group and in the "DQRF" control group. FSDS-R basal mean scores more than halved in the active group $(-57.3 \%, \mathrm{p}<0.01)$; the women of the "DQRF" control group showed a tendency towards a somewhat less dramatic improvement of sexual distress $(-49.6 \%$ vs. mean basal score, $\mathrm{p}<0.01$ ), with a marginally significant difference between the two treatment groups $(\mathrm{p} \approx 0.054)$.

Figure 3 illustrates the women's experience with vaginal intercourse and how the related sexual satisfaction evolved in the "DQRF" $+\mathrm{UPR}^{\mathrm{m} \text { "s" }}$ active group and in the "DQRF ${ }^{\text {man" }}$ control women. The improvement of SSQ mean scores was highly significant in both treatment groups ( $+96.4 \%$ and $+85.8 \%$, respectively; $\mathrm{p}<0.01$ vs. mean basal score for both groups), but the final SSQ improvement was significantly higher in the "DQRF" ${ }^{\mathrm{Tm}}+\mathrm{UPR}^{\mathrm{m} \mathrm{m} \text { ” }}$ treatment group $(\mathrm{p}<0.05)$.

\section{Discussion}

Radiofrequency fields induce oscillating electrical currents in target tissues with steady re-orientation of dipole moments like water molecules. Water viscosity means resistance (impedance) and attrition to movements of other biomolecules under the influence of the variable electrical fields in female intimate tissues. That results in dissipation of biomolecular kinetic energy into heat [2]. More and more accumulating evidences suggest that the DQRF ${ }^{\mathrm{mi}}$ technology could be an advance, most likely in terms of safety, over other light- and energybased technologies. Laser devices may especially cause bleeding, pain, 


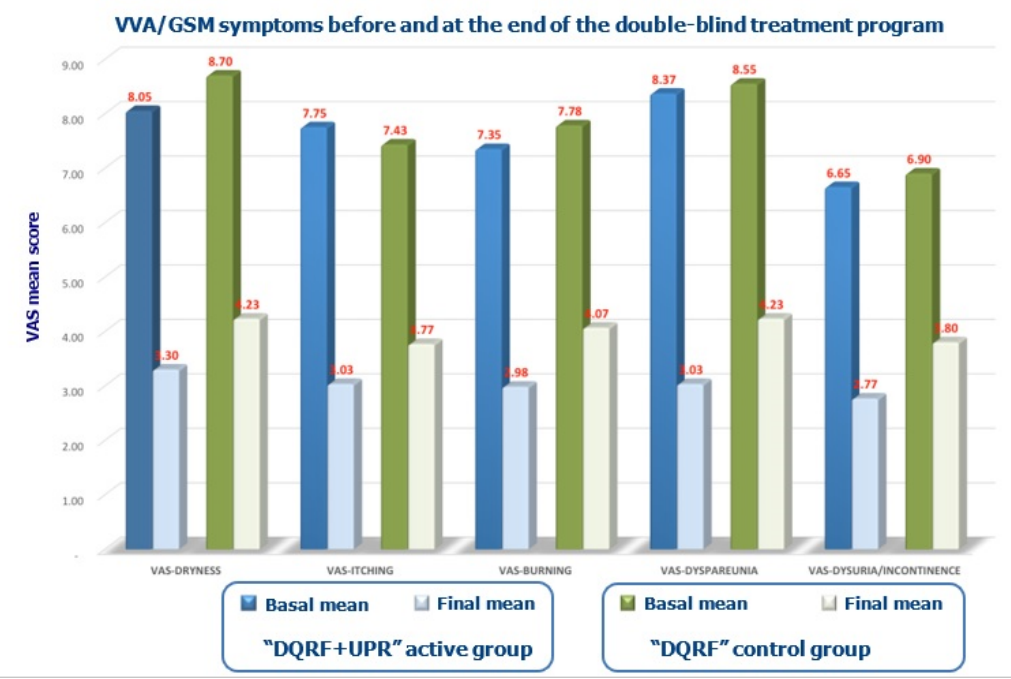

Figure 2. FSDS-R mean scores (main factors affecting the women's personal distress due to sexual dysfunction) in the active "DQRFTM + UPR control "DQRFTM" treatment group

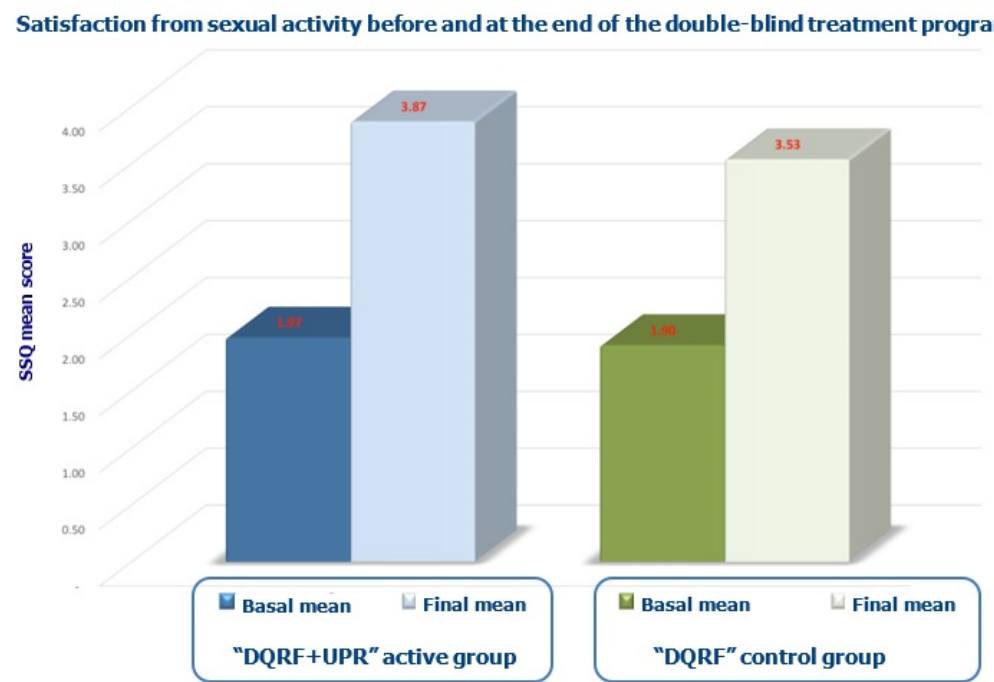

Figure 3. SSQ mean scores (sexual satisfaction from vaginal intercourse) in the active "DQRFTM + UPR

and burning [14]. Induction of new elastogenesis is also relatively unique to radiofrequency technologies, helping to restore mechanical strength, tightness, and elasticity to atrophic external genitalia of postmenopausal women $[2,15,16]$.

The novel UPR ${ }^{\text {Tx }}$ technology was devised to modify the performance of the DQRF ${ }^{\text {rx }}$ EVA $^{\text {six }}$ device so as to facilitate the penetration of any topical active principle applied to the treated vulvo-vaginal area. The idea behind the $\mathrm{UPR}^{\mathrm{ru}}$ concept is to enhance the established

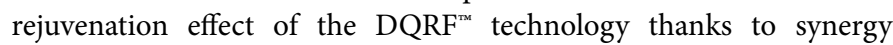
between the biological effect of the radiofrequency treatment and that of the topical agents that were shown to improve the atrophy of female post-menopausal intimate tissues. Available evidence led to choose lowmolecular weight $\mathrm{HA}$ as the model topical active principle to test the $\mathrm{UPR}^{\mathrm{Tw}}$ concept in the current short-term pilot study [9-12]. The DQRF ${ }^{\mathrm{mw}}$ technology has once again confirmed its rapid efficacy on the often troubling VVA/GSM symptoms as well as on other problems relating to the woman's sexual life and self-esteem. Outcomes were in line with those observed in previous studies in post-menopausal women.
A general tendency was apparent in the "DQRF ${ }^{\mathrm{rm} n}$ treatment group to about halve the severity of symptoms and the disruption of intimate life over the about two months of the five-session treatment program $[1,3]$. The $\mathrm{UPR}^{\mathrm{m}}$ concept tested in this double-blind pilot study also seems validated thanks to a global enhancement of the DQRF ${ }^{m}$ clinical benefits with special reference to VVA/GSM symptoms like vaginal itching and dysuria/incontinence. Interestingly, the score differences between the "DQRF" $+\mathrm{UPR}^{\mathrm{mw} \text { " }}$ active group and the "DQRF" control women that support the UPR ${ }^{\mathrm{ix}}$ concept were especially strong in the area of the women's everyday sexual life: dyspareunia, distress related to sexual dysfunction, gratification directly related to sexual activity. Of course, long-term studies are warranted to confirm these preliminary encouraging results.

\section{Conflicts of interest}

The authors were in the past consultants to Novavision Group S.p.A. They certify to have no current conflict of interest with any financial or commercial organization regarding the content of this manuscript. 


\section{References}

1. Vicariotto F, De Seta F, Faoro V, Raichi M (2017) Dynamic quadripolar radiofrequency treatment of vaginal laxity/menopausal vulvo-vaginal atrophy: 12-month efficacy and safety. Minerva Ginecol 69: 342-349. [Crossref]

2. Tadir Y, Gaspar A, Lev-Sagie A, Alexiades M, Alinsod R, et al (2017) Light and energy based therapeutics for genitourinary syndrome of menopause: consensus and controversies. Lasers Surg Med 49: 137-159. [Crossref]

3. Vicariotto F, Raichi M (2016) Technological evolution in the radiofrequency treatment of vaginal laxity and menopausal vulvo-vaginal atrophy and other genitourinary symptoms: first experiences with a novel dynamic quadripolar device. Minerva Ginecol 68: 225-236. [Crossref]

4. Zhan Y, Cao Z, Bao N, Li J, Wang J, et al (2012) Low-frequency ac electroporation shows strong frequency dependence and yields comparable transfection results to dc electroporation. J Control Release 160: 570-576. [Crossref]

5. Zald PB, Cotter MA 2nd, Robertson ES (2000) Improved transfection efficiency of 293 cells by radio frequency electroporation. Biotechniques 28: 418-420. [Crossref]

6. Landau M, Fagien S (2015) Science of hyaluronic acid beyond filling: fibroblasts and their response to the extracellular matrix. Plast Reconstr Surg 136(5 Suppl): 188S-195S. [Crossref]

7. Fasola E, Gazzola R (2016) Labia majora augmentation with hyaluronic acid filler: technique and results. Aesthet Surg J 36: 1155-1163. [Crossref]

8. Zerbinati N, Haddad RG, Bader A, Rauso R, D'Este E, et al (2017) A new hyaluronic acid polymer in the augmentation and restoration of labia majora. J Biol Regul Homeost Agents 31(2 Suppl. 2): 153-161. [Crossref]
9. Stute $\mathrm{P}$ (2013) Is vaginal hyaluronic acid as effective as vaginal estriol for vaginal dryness relief? Arch Gynecol Obstet 288: 1199-1201. [Crossref]

10. Origoni M, Cimmino C, Carminati G, Iachini E, Stefani C, et al (2016) Postmenopausal vulvovaginal atrophy (VVA) is positively improved by topical hyaluronic acid application. A prospective, observational study. Eur Rev Med Pharmacol Sci 20: 41904195. [Crossref]

11. Jokar A, Davari T2, Asadi N, Ahmadi F, Foruhari S (2016) Comparison of the hyaluronic acid vaginal cream and conjugated estrogen used in treatment of vaginal atrophy of menopause women: a randomized controlled clinical trial. Int J Community Based Nurs Midwifery 4: 69-78. [Crossref]

12. Chen J, Geng L, Song X, Li H, Giordan N, et al (2013) Evaluation of the efficacy and safety of hyaluronic acid vaginal gel to ease vaginal dryness: a multicenter, randomized, controlled, open-label, parallel-group, clinical trial. J Sex Med 10: 15751584. [Crossref]

13. Fisher TD, Davis CM, Yarber WL \& Davis S L. Handbook of Sexuality-Related Measures, 3rd edition. Routledge, New York, 2013.

14. Gaspar A, Addamo G, Brandi $\mathrm{H}$ (2011) Vaginal fractional $\mathrm{CO}_{2}$ laser: a minimally invasive option for vaginal rejuvenation. Am J Cosmetic Surg 28: 156-162.

15. Beasley KL, Weiss RA (2014) Radiofrequency in cosmetic dermatology. Dermatol Clin 32: 79-90. [Crossref]

16. Alexiades M, Berube D (2015) Randomized, blinded, 3-arm clinical trial assessing optimal temperature and duration for treatment with minimally invasive fractional radiofrequency. Dermatol Surg 41: 623-632. [Crossref]

Copyright: $₫ 2018$ Tranchini R. This is an open-access article distributed under the terms of the Creative Commons Attribution License, which permits unrestricted use, distribution, and reproduction in any medium, provided the original author and source are credited. 